Pacific Journal of Mathematics

RINGS WHERE THE ANNIHILATORS OF $\alpha$-CRITICAL 


\title{
RINGS WHERE THE ANNIHILATORS OF $\alpha$-CRITICAL MODULES ARE PRIME IDEALS
}

\author{
E. H. FeLleR
}

For a ring $R$ with Krull dimension $\alpha$, we investigate the property that the annihilators of $\alpha$-critical modules are prime ideals. If $R$ satisfies the large condition then this property holds iff $R / I_{0}$ is semiprime, where $I_{0}$ is the maximal right ideal of Krull dimension $<\alpha$. The property holds in the following rings, (i) $R$ is weakly ideal invariant, (ii) $R$ satisfies the left AR property, or (iii) the prime ideals of $R$ are right localizable. In addition, if $R$ is a hereditary Noetherian $\alpha$-primitive ring, then $R$ is a prime ring.

1.1. Introduction. This paper will provide conditions on a ring $R$ with Krull dimension $\alpha$, which imply the property that the annihilator of any $\alpha$-critical module is a prime ideal. In the terminology of [2], this property means the $\alpha$-coprimitive ideals are prime.

In $\S 2$, using the procedures of [2] and [4], we find necessary and sufficient conditions for this property in rings which satisfy the large condition. In addition, for a ring $R$ with Krull dimension this property is true under any one of the following conditions; (i) $R$ is weakly ideal invariant (ii) $R$ satisfies the left AR-condition, (iii) the prime ideals of $R$ are right localizable. For right Noetherian ring, the conditions (i) and (iii) are shown to imply this property in [5]. For Noetherian AR-rings the same is true from [5] and [13]. We extend the results of K. Brown, T. H. Lenagan, and J. T. Stafford [5] for (i), (ii), and (iii) to rings with Krull dimension. The proofs are short and direct, utilizing the procedures of [2] and [4]. This should be helpful in the study of related problems.

One can show directly that if a right Noetherian ring $R$ is smooth and the $\alpha$-coprimitive ideals are prime, then $R$ has a right Artinian, right classical quotient ring.

In $\S 3$, we shall investigate right hereditary $\alpha$-primitive rings $R$. We show that the associated $\alpha$-prime ideal $P$ is a direct summand, and $R / P$ is a right hereditary prime ring. This implies from [6] and [11], that if $R$ is a hereditary Noetherian $\alpha$-primitive ring, then $R$ is a hereditary Noetherian prime ring of Krull dimension 0 or 1 .

All rings will have identity, and all modules are right unitary modules. Ideal shall mean two-sided ideal, and a ring is Noetherian if it is both right and left Noetherian. The injective hull of a 
module $M$ is denoted by $E(M)$, and $|M|$ denotes the Krull dimension of $M$.

If $S$ is a subset of a module $M$ over $R$, then ann $S=S^{r}=$ $\{x \in R \mid s x=0$ for all $s \in S\}$. The Socle of $M$ is the sum of the critical submodules of $M$, and is denoted by Soc $M$.

2.1. What $\alpha$-coprimitive ideals are prime. As in [2], an ideal $D$ of $R$ is called $\alpha$-coprimitive if $D$ is the annihilator of an $\alpha$-critical module $C$, where $|C|=|R|=\alpha$. A ring $R$ is $\alpha$-primitive provided 0 is an $\alpha$-coprimitive ideal. If $I$ is an indecomposable injective module containing an $\alpha$-critical module, then $I$ is called an $\alpha$ indecomposable injective module.

The following is known and the proof direct.

Proposition 2.2. If $R$ is a semiprime ring, where $|R|=\alpha$, then every $\alpha$-coprimitive ideal is prime.

From $\S 2$ of [4], if $|R|=\alpha$, for every $\alpha$-indecomposable module $I$, there is a unique minimal $\alpha$-coprimitive ideal $D$, such that $D=$ ann Soc $I$, and if $C$ is any $\alpha$-critical module in $I$, then $D \subseteq$ ann $I \leqq P$, where $P=$ ass $I$. Thus we can write.

Proposition 2.3. If $|R|=\alpha$, then every $\alpha$-coprimitive ideal is prime iff for every $\alpha$-indecomposable injective module $I$, we have ann (Soc I) is prime ideal.

Since there is but a finite number of isomorphic classes of $\alpha$ indecomposable injective modules, then from 2.2 and 2.3 we have.

Proposition 2.4. If $|R|=\alpha$, and $M=I_{1} \oplus \cdots \oplus I_{n}$, where $I_{i}$, for $i=1,2, \cdots, n$, is an $\alpha$-indecomposable injective, and each isomorphic class of $\alpha$-indecomposable injective modules is represented in this sum, then every $\alpha$-coprimitive ideal is prime iff ann (Soc $M$ ) is a semiprime ideal of $R$.

A ring $R$ with Krull dimension $\alpha$ is said to satisfy the large condition, provided $|R / L|<|R|$, for all large right ideals $L$ of $R$. A ring $R$ is $\alpha$-smooth or $\alpha$-homogeneous if $|K|=|R|=\alpha$, for all nonzero right ideals $K$ of $R$.

Proposition 2.5. Let $R$ be an $\alpha$-smooth ring with Krull dimension $\alpha$. Then $R$ satisfies the large condition and every $\alpha$ coprimitive ideal is prime iff $R$ is a semiprime ring. 
Proof. If $R$ satisfies the large condition, then every $\alpha$ indecomposable injective module embeds in $E(R)$. Hence, since $R$ is smooth, then $R \cong I_{1} \oplus \cdots \oplus I_{n}$, where each $I_{i}$ is an $\alpha$-indecomposable injective module, and all the isomorphic classes are represented. From Corollary 2.6 of $\left[2\right.$, p. 61], we have Soc $I_{i}=I_{i}$ for all $i$. Hence $\operatorname{Soc} E(R)=E(R)$. Now if every $\alpha$-coprimitive ideal is prime, then from 2.4, we have $0=$ ann $R=$ ann $E(R)$ is a semiprime ideal.

Since semiprime rings with Krull dimension all satisfy the large condition the converse is true.

THEOREM 2.6. Let $R$ be a right Noetherian ring with Krull dimension $\alpha$, then $R$ satisfies the large condition and the $\alpha$-coprimitive ideals are prime iff $I_{0}$ is a closed semiprime ideal, where $I_{0}$ is the maximal right ideal of Krull dimension $<\alpha$.

Proof. If $D$ is an $\alpha$-coprimitive ideal of $R$, then since $R / D$ is $\alpha$-smooth, it follows that $D \supseteqq I_{0}$, which is an ideal of $R$. Thus the $\alpha$-coprimitive ideals of $R / I_{0}$ are just of the form $D / I_{0}$, where $D$ is an $\alpha$-coprimitive ideal of $R$.

From Proposition 3.4 of [3], we know that $R$ satisfies the large condition iff $I_{0}$ is closed and $R / I_{0}$ satisfies the large condition. Since $R / I_{0}$ is smooth, the result follows from 2.5.

ExAMPLE 2.7. Let $Z$ denote the integers and $Z_{p}$ the integers modulo a prime element $p$. If

$$
R_{1}=\left[\begin{array}{cc}
Z & Z_{p} \\
0 & Z_{p}
\end{array}\right] \text { and } R_{2}=\left[\begin{array}{cc}
Z & Z_{p}[x] \\
0 & Z_{p}[x]
\end{array}\right],
$$

then $R_{1}$ and $R_{2}$ satisfy the large condition, and $R_{1}$ satisfies the conditions of 2.6. However, $R_{2}$ is smooth, but certainly not semiprime. The ideal $\left[\begin{array}{ll}(p) & 0 \\ 0 & 0\end{array}\right]$ is coprimitive, and not prime.

If $R$ is a ring with Krull dimension, an ideal $T$ of $R$ is said to be weakly ideal invariant provided $|T / I T|<|R / T|$ for every right ideal $I$ of $R$ with $|R / I|<|R / T|$. If every ideal of $R$ is weakly ideal invariant, then $R$ is said to be weakly ideal invariant.

The proof of the following is direct.

LEMMA 2.8. If $A, B$, and $C$ are right ideals of a ring with Krull dimension $\alpha$ and if $|A / B|=\alpha$ and $|R / C|<\alpha$, then $|A \cap C / B \cap C|=\alpha$.

THEOREM 2.9. Let $R$ be a ring where $|R|=\alpha$. If $N$, the prime 
radical of $R$, is weakly ideal invariant, then the $\alpha$-coprimitive ideals are prime.

Proof. Let $N=P_{1} \cap \cdots \cap P_{m} \cap P_{m+1} \cap \cdots \cap P_{n}$, where the $P_{i}$ are minimal prime ideals, and $\left|R / P_{i}\right|=\alpha$ for $i=1,2, \cdots, m$, and $\left|R / P_{i}\right|<\alpha$ for $i=m+1, \cdots, n$.

As in [4], let $D_{i}$ be the unique minimal $\alpha$-coprimitive ideal contained in $P_{i}$ for $i=1, \cdots, m$, and $H_{i}=\left(D_{i}: P_{i}\right)=\left\{x \in R \mid x P_{i} \leqq D_{i}\right\}$ for $i=1,2, \cdots, m$. Then $H_{i} / D_{i}$ is large in $R / D_{i}$, since $P_{i} / D_{i}$ is the assassinator for every uniform right ideal of $R / D_{i}$. From [2], we have that $R / D_{i}$ satisfies the large condition. Hence $\left|R / H_{i}\right|<\alpha$. From Corollary 1.3 of [9], for rings will Krull dimension, if $W=$ $H_{1} \cdots H_{m} P_{m+1} \cdots P_{n}$, then $|R / W|<\alpha$. Now $W N \subset D_{1} \cap \cdots \cap D_{m} \cap$ $P_{m+1} \cap \cdots \cap P_{n}$. If $D_{i} \neq P_{i}$ for some $i$, then $\left|P_{1} \cap \cdots \cap P_{m}\right| D_{1} \cap \cdots \cap D_{m} \mid=$ $\alpha$ since $R / D_{1} \cap \cdots \cap D_{m}$ is $\alpha$-smooth, and $P_{1} \cap \cdots \cap P_{m}$ is not contained in $D_{1} \cap \cdots \cap D_{m}$. To show this last statement, suppose $P_{1} \cap \cdots \cap P_{m}=$ $D_{1} \cap \cdots \cap D_{m}$. Then $P_{i}\left(P_{1} \cdots P_{i-1} P_{i+1} \cdots P_{m}\right) \subseteq D_{i}$. However, $D_{i}$ is $P_{i}$ primary, which means $P_{1} \cdots P_{i-1} P_{i+1} \cdots P_{m} \subseteq P_{i}$, and $P_{j} \subseteq P_{i}$ for $i \neq j$, which is a contradiction.

Thus, by Lemma 2.8, we have $|N / W N|=\alpha$. However $|R / W|<\alpha$, which contradicts the assumption that $N$ is weak ideal invariant.

It is not known whether the converse of this theorem is true for rings with Krull dimension. However, Theorem 2.5 of [5] establishes this theorem and its converse for right Noetherian rings.

If $I$ is an ideal of ring $R$, and $C(I)=\{c \in R \mid c+I$ is regular in $R / I\}$, then $I$ is said to be right localizable provided $C(I)$ is a right Ore set.

THEOREM 2.10. Let $R$ be an $\alpha$-primitive ring with unique $\alpha$ prime ideal $P$, then $P$ is right localizable iff $P=0$.

Proof. If $P=0$, the result follows from [7]. Suppose now $P \neq 0$, and $P$ is right localizable. Then $[0: C(P)]=\{x \in R \mid x a=0$ for some $a \in C(P)\}$ is an ideal of $R$, and $[0: C(P)] \subseteq P$.

Suppose $[0: C(P)]=P$. Now $P^{l}$, the left annihilator of $P$, is a large right ideal of $R$, hence $P^{l} \cap P \neq 0$. There exists $a \in C(P)$, and $0 \neq x \in P^{l} \cap P$, so that $x(a R+P)=0$. However, $|R / a R+P|<\alpha$, which implies $|x R|<\alpha$. This is not possible since $R$ is $\alpha$-smooth. Hence [0: $C(P)] \varsubsetneqq P$.

Since $P^{l}$ is not contained in $P$, then $P^{l} \cap C(P) \neq 0$. Let $a \in P^{l} \cap$ $C(P)$, and $x \in P$, but $x \notin[0: C(P)]$. By the Ore condition, there exist, $d \in R, b \in C(P)$ such that $a d=x b$. Thus $d \in P$, and since $a \in P^{l}$, then $a s=0$. This implies $x b=0$ and $x \in[0: C(P)]$, a contradiction.

Note that if $P$ is right localizable in $R$, then $P / K$ is right 
localizable in $R / K$ for any ideal $K$ contained in $P$. Thus

CoRollary 2.11. If $R$ is a ring with $|R|=\alpha$, and if every $\alpha$ prime $P$ is right localizable, then the $\alpha$-coprimitive ideals are prime.

For right Noetherian rings this result follows from 2.5 and 3.1 of [5].

An ideal $I$ of a ring $R$ is said to satisfy the left AR property provided for every left ideal $E$ of $R$, there exists a positive integer $n$ such that $E \cap I^{n} \subseteq I E$. A ring $R$ satisfies the left AR property if every ideal of $R$ satisfies this property. The right AR property is defined in a similar fashion.

THEOREM 2.12. If $R$ is an $\alpha$-primitive ring with unique $\alpha$ prime ideal $P$, then $P=0$ iff $P^{l}$ satisfies the left AR property.

Proof. If $P=0$, the result is trivial. Suppose $P^{l}$ satisfies the left AR property. Since $P^{l}$ is large, and $Z(R)=0$, then $\left(P^{l}\right)^{n}$ is large for all positive integer $n$. Thus, if $P \neq 0$, then $0 \neq P \cap\left(P^{l}\right)^{n} \subseteq$ $P^{l} P=0$, a contradiction.

Note that if $I$ satisfies the left AR-condition in $R$, then $I / K$ satisfies this condition in $R / K$ for all ideals $K$ contained in $I$.

CoROLlaRy 2.13. If a ring $R$ with Krull dimension satisfies the left AR property, then the $\alpha$-coprimitive ideals are prime.

If $R$ is a Noetherian ring with both the right and left ARcondition, the result follows from 3.4 of [5], which is a consequence of 3.4 of [13].

Proposition 2.14. Let $R$ be an $\alpha$-coprimitive ring with unique $\alpha$-prime $P$, then $P$ is nilpotent iff $P$ satisfies the right AR-condition.

Proof. Now $P^{l} \cap P^{n} \cong P^{l} P=0$ for some positive integer $n$. Since $P^{l}$ is large, we have $P^{n}=0$.

For an example of this type of ring see 4.3 of [4].

3.1. Right hereditary $\alpha$-primitive rings. Currently, we have no example of a Noetherian $\alpha$-primitive ring $R$, which is not prime. If $\left.\right|_{R} M|=| M_{R} \mid$ for all $(R, R)$ modules $M$, one easily shows $R$ is prime. Thus an example would likely depend on finding a Noetherian ring, whose right Krull dimension is not equal to its left Krull dimension. 
We show here that a hereditary Noetherian $\alpha$-primitive ring is prime. We begin with an investigation of right hereditary $\alpha$ primitive rings.

Proposition 3.2. Let $R$ be a right hereditary $\alpha$-primitive ring with faithful $\alpha$-critical module $C$. Then

(1) If $K$ is a right of $R$, then $K^{r}$ is a direct summand of $R$.

(2) The ass $C=P$ is a direct summand of $R$, and $R / P$ is a right hereditary ring.

(3) $P^{r}=0$, and $R$ is right Noetherian.

Proof. Since $R$ is smooth, then for a right ideal $K$ of $R$, we have $K^{r}=x_{1}^{r} \cap \cdots \cap x_{n}^{r}$, for $x_{1}, x_{2}, \cdots, x_{n} \in K$. Thus $R / K$ imbeds in $R / x_{1}^{r} \oplus \cdots \oplus R / x_{n}^{r}$, and by Proposition 7 of $[10$, p. 85], we have that $R / K$ is projective. Hence $K$ is a direct summand of $R$.

If $C_{0}$ is a compressible right ideal of $R$, then $C_{0}^{r}=P$ from [2]. Thus (2) follows from (1).

Since $R$ is $P$ primary, then $P^{r} \subseteq P$. Thus $\left(P^{r}\right)^{2}=0$. If $P^{r} \neq 0$, then (1) implies that $P^{r}$ contains a nonzero idempotent, which is impossible. The ring $R$ is right Noetherian by Corollary 5.20 of [8, p. 149].

Since $P$ is a direct summand of $R$, then $R=e R \oplus P$, where $(1-e) R=P$. We can write $R$ as a formal triangular matrix ring.

$$
R \cong\left(\begin{array}{cc}
(1-e) R(1-e) & (1-e) R e \\
0 & e R
\end{array}\right),
$$

where

$$
P=\left(\begin{array}{cc}
(1-e) R(1-e) & (1-e) R e \\
0 & 0
\end{array}\right)
$$

and

$$
R / P \cong\left(\begin{array}{rr}
0 & 0 \\
0 & e R
\end{array}\right)
$$

is a right hereditary, right Noetherian prime ring, and $(1-e) R(1-e)=$ $(1-e) P(1-e) \cong \operatorname{Hom}_{R}(P, P)$ is a right hereditary ring. Theorem 4.7 of $[8, p$. 111] provides a characterization of triangular matrix rings of this type.

If $P \neq 0$, these rings do not satisfy the left or the right ARcondition. Thus if $R$ is a right hereditary $\alpha$-primitive ring which satisfies the right AR-condition, then $R$ is a prime ring.

THEOREM 3.3. If $R$ is a Noetherian right hereditary $\alpha$-primitive ring, then $R$ is a hereditary Noetherian prime ring of Krull dimension 0 or 1. 
Proof. We have $R / P$ is a right hereditary prime ring, and by Theorem 3 of [12], then $R / P$ is a hereditary Noetherian prime ring. Consequently, by 3.52 of $[6$, p. 310], then $|R / P|=0$ or 1 . Since $|R|=|R / P|$, then $|R|=0$ or 1 . If $|R|=0$, then the faithful critical module $C$ is simple. If $|R|=1$, the result follows from Lemma 3.5 of [11].

From 2.3, we have

CoROLLARY 3.4. Let $R$ is a Noetherian ring of Krull dimension $\alpha$ and $I$ denote an $\alpha$-indecomposable injective module. If $R /$ ann Soc $I$ is right hereditary for all $I$, then for every $\alpha$-coprimitive ideal $D$ we have $R / D$ is a hereditary prime ring. If $\alpha=|R|$, then $\alpha=0$ or 1.

The upper triangular matrices over $F[x]$, where $F$ is a field, is an example for this corollary.

Proposition 3.5. Let $R$ be a right Noetherian $\alpha$-primitive ring with faithful projective $\alpha$-critical module $C$. Then $R$ is right hereditary iff $C$ is hereditary. In this case, $R$ is a direct sum of critical right ideal, at least one of which is faithful.

Proof. If $C$ is projective, and $R$ is right hereditary certainly $C$ is hereditary. If $C$ is hereditary, then as in [2], there exists $x_{1}, x_{2}, \cdots, x_{n} \in C$, such that $x_{1}^{r} \cap \cdots \cap x_{n}^{r}=0$. As in 3.2, then $R$ is right hereditary, and is a direct sum of critical right ideals.

If $C$ is projective, then $C$ embeds in direct sum of copies of the right hereditary ring $R$. Again by Proposition 7 of $[10$, p. 85], since $C$ is critical, then $C$ embeds in $R$. If $R=\sum_{i=1}^{n} C_{i}$, where $C_{i}$ is a critical right ideal for each $i$, then $C_{i} C \neq 0$ for some $i$. Hence there exist a monomorphism of $C \rightarrow C_{i}$. Thus $C_{i}$ is faithful, and the proof is complete.

CoROLlary 3.6. Let $R$ be a Noetherian ring of Krull dimension $\alpha$, where all the $\alpha$-indecomposable injective modules $I$ are semihereditary. If $D$ is the ann Soc $I$, then $R / D$ is a hereditary prime ring, and the $\alpha$-coprimitive ideals are prime.

The $\alpha$-primitive rings which are the direct sum of critical right ideals, at least one of which is faithful, is described in [1].

Example 6.6. Let $F$ be a field, and $F[x]$ the polynomial ring in $x$ over $F$. Let 


$$
R_{1}=\left|\begin{array}{ccc}
F & F & F[x] \\
0 & F & F[x] \\
0 & 0 & F[x]
\end{array}\right|, \quad \text { and } \quad R_{2}=\left|\begin{array}{ccc}
F & 0 & F[x] \\
0 & F & F[x] \\
0 & 0 & F[x]
\end{array}\right|
$$

Then $R_{1}$ and $R_{2}$ have faithful a critical module $C=\left|\begin{array}{ccc}F & F & F[x] \\ 0 & 0 & 0 \\ 0 & 0 & 0\end{array}\right|$. Now $R_{1}$ and $R_{2}$ are right hereditary, by Theorem 4.7 of [8, p. 111]. Now $C$ is hereditary over $R_{1}$, but $C$ does not embed in $R_{2}$. Hence $C$ is not projective over $R_{2}$.

\section{REFERENCES}

1. A. K. Boyle, M. G. Deshpande and E. H. Feller, On nonsingularly k-primitive rings, Pacific J. Math., 68 (1977), 303-311.

2. A. K. Boyle and E. H. Feller, Semicritical modules and k-primitive rings, Module theory, Springer-Verlag Lecture Notes, No. 700 (1979).

3. A. K. Boyle, The large condition for rings with Krull dimension, Proc. Amer. Math. Soc., 72 (1),, (Oct. 1978), 27-32.

4. E. H. Feller and A. K. Boyle, $\alpha$-indecomposable injective modules and $\alpha$-coprimitive ideals, Comm. in Alg., 8 (12) (1980), 1151-1167.

5. K. A. Brown, T. H. Lenagan and J. T. Stafford, Weak ideal invariance and localization, (to appear).

6. A. W. Goldie, The structure of Noetherian rings, Springer-Verlag Lecture notes, No. 246, (1972).

7. R. Goldon and J. C. Robson, Krull Dimension, Amer. Math. Soc. Memoirs, No. 133, (1973).

8. K. R. Goodearl, Ring Theory, Marcel Dekker, New York, 1976.

9. A. V. Jategankar, Jacobsons Conjecture and modules over fully bounded Noetherian rings, J. Algebra, 30 (1974), 103-121.

10. J. Lambek, Lectures on Rings and Modules, Chelsea Pub. Co., 1976.

11. T. H. Lenagan, Neotherian rings with Krull dimension one, J. London Math. Soc., 15 (1977), 41-47.

12. L. W. Small, Semi-hereditary Rings, Bull. Amer. Math. Soc., 73 (1967), 656-658.

13. P. F. Smith, Localization and the AR-property, Proc. London Math. Soc. (3), 22 (1971).

Received March 5, 1980.

ThE UNIVERSITY OF WISCONSIN

Milwaukee, WI 53201 


\title{
PACIFIC JOURNAL OF MATHEMATICS
}

\section{EDITORS}

\author{
DoNALD BABBITT (Managing Editor) \\ University of California \\ Los Angeles, CA 90024 \\ Hugo RossI \\ University of Utah \\ Salt Lake City, UT 84112 \\ C. C. MOORE and ANDrew OGG \\ University of California \\ Berkeley, CA 94720
}

J. DugundJI

Department of Mathematics

University of Southern California

Los Angeles, CA 90007

R. FINN and J. MILGRAM

Stanford University

Stanford, CA 94305

\section{ASSOCIATE EDITORS}
R. Arens
E. F. BeCKenbaCH
B. H. NeumanN
F. WOLF
K. YoSHIDA

\section{SUPPORTING INSTITUTIONS}

UNIVERSITY OF ARIZONA

UNIVERSITY OF BRITISH COLUMBIA

CALIFORNIA INSTITUTE OF TECHNOLOGY

UNIVERSITY OF CALIFORNIA

MONTANA STATE UNIVERSITY

UNIVERSITY OF NEVADA, RENO

NEW MEXICO STATE UNIVERSITY

OREGON STATE UNIVERSITY

\author{
UNIVERSITY OF OREGON \\ UNIVERSITY OF SOUTHERN CALIFORNIA \\ STANFORD UNIVERSITY \\ UNIVERSITY OF HAWAII \\ UNIVERSITY OF TOKYO \\ UNIVERSITY OF UTAH \\ WASHINGTON STATE UNIVERSITY \\ UNIVERSITY OF WASHINGTON
}

The Supporting Institutions listed above contribute to the cost of publication of this Journal, but they are not owners or publishers and have no responsibility for its content or policies.

Mathematical papers intended for publication in the Pacific Journal of Mathematics should be in typed form or offset-reproduced, (not dittoed), double spaced with large margins. Please do not use built up fractions in the text of the manuscript. However, you may use them in the displayed equations. Underline Greek letters in red, German in green, and script in blue. The first paragraph or two must be capable of being used separately as a synopsis of the entire paper. Please propose a heading for the odd numbered pages of less than 35 characters. Manuscripts, in triplicate, may be sent to any one of the editors. Please classify according to the scheme of Math. Reviews, Index to Vol. 39. Supply name and address of author to whom proofs should be sent. All other communications should be addressed to the managing editor, or Elaine Barth, University of California, Los Angeles, California, 90024.

50 reprints to each author are provided free for each article, only if page charges have been substantially paid. Additional copies may be obtained at cost in multiples of 50 .

The Pacific Journal of Mathematics is issued monthly as of January 1966. Regular subscription rate: $\$ 102.00$ a year $(6$ Vols., 12 issues). Special rate: $\$ 51.00$ a year to individual members of supporting institutions.

Subscriptions, orders for numbers issued in the last three calendar years, and changes of address shoud be sent to Pacific Journal of Mathematics, P.O. Box 969, Carmel Valley, CA 93924, U.S.A Old back numbers obtainable from Kraus Periodicals Co., Route 100, Millwood, NY 10546.

\section{PUBLISHED BY PACIFIC JOURNAL OF MATHEMATICS, A NON-PROFIT CORPORATION}

Printed at Kokusai Bunken Insatsusha (International Academic Printing Co., Ltd.). 8-8, 3-chome, Takadanobaba, Shinjuku-ku, Tokyo 160, Japan. 


\section{Pacific Journal of Mathematics}

Vol. 93, No. $2 \quad$ April, 1981

Ilya Eugene Blum and Srinivasa Swaminathan, Continuous selections and

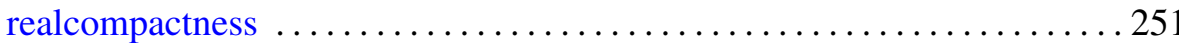

Lawrence James Brenton, Differentiably $k$-normal analytic spaces and extensions of holomorphic differential forms $\ldots \ldots \ldots \ldots \ldots \ldots \ldots 261$

Jo-Ann Deborah Cohen, Topologies on the ring of integers of a global field ................................................... 269

Robert Jay Daverman, Detecting the disjoint disks property $\ldots \ldots \ldots \ldots 277$

Edmund H. Feller, Rings where the annihilators of $\alpha$-critical modules are prime ideals ....................................... 299

Richard Elam Heisey and Henryk Torunczyk, On the topology of direct

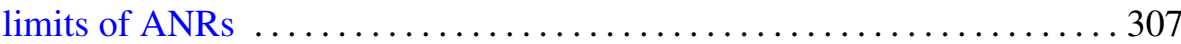

Gerald William Johnson and David Lee Skoug, Notes on the Feynman

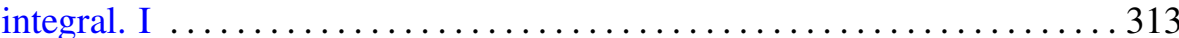

Michael S. Keane and Stuart Jay Sidney, Distinguishing a plane curve

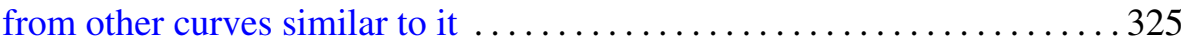

Leonid A. Luxemburg, On compact metric spaces with noncoinciding

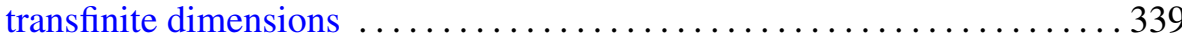

Chun Ming Ma, A uniqueness theorem for Navier-Stokes equations . . . . . . 387

Donald J. Newman and Theodore Joseph Rivlin, A characterization of the weights in a divided difference . . . . . . . . . . . . . . . . . . . 407

Marc Aristide Rieffel, $C^{*}$-algebras associated with irrational rotations $\ldots .415$

Kichi-Suke Saito, Invariant subspaces for finite maximal subdiagonal algebras

Frederic W. Shultz, Dual maps of Jordan homomorphisms and ${ }^{*}$-homomorphisms between $C^{*}$-algebras $\ldots \ldots \ldots \ldots$

Vsevolod Alekseevich Solonnikov, On the solvability of boundary and initial-boundary value problems for the Navier-Stokes system in domains with noncompact boundaries

Tavan Thomas Trent, New conditions for subnormality

L. E. Ward, Extending Whitney maps ..................

Leslie Wilson, Jets with regular zeros

Sergio Eduardo Zarantonello, The sheaf of $H^{p}$-functions in product 